\title{
SAÚdE ESCOLAR: $O$ ELO DE UNIÃO ENTRE SAÚdE E EDUCAÇÃO
}

\author{
SCHOOL HEALTH: THE BOND BETWEEN HEALTH AND EDUCATION \\ SALUD ESCOLAR: EL PUNTO DE UNIÓN ENTRE SALUD Y EDUCACIÓN
}

António Reis do Arco*

\begin{abstract}
RESUMO:
Objetivo: Refletir sobre a importância da saúde escolar na promoção da saúde coletiva. Método: Reflexão teórica baseada na revisão da literatura. Resultados: As crianças e adolescentes necessitam de ser saudáveis para aprender e de ser educados para serem saudáveis, pelo que a saúde e a educação devem atuar em conjunto, visando proporcionar uma resposta adequada às necessidades de saúde e educação das populações. Perante os problemas quotidianos que ameaçam a saúde e o bem-estar, pode-se considerar a importância desta colaboração, representando a saúde escolar um instrumento que engloba não só o sistema de saúde e de educação, como outros intervenientes, tais como as forças de segurança, a sociedade civil, a comunidade e a familia. Conclusões: Este é um dos desafios que se coloca atualmente às organizações escolares e de saúde, assegurar uma relação eficaz com o contexto social em que se inserem, participando e incrementando programas de saúde escolar que respondam às reais necessidades da comunidade.

Palavras-chave: Saúde escolar, Promoção da saúde, Equidade em saúde, Educação, Participação comunitária.
\end{abstract}

\begin{abstract}
:
Objective: To reflect on the importance of health education in health promotion. Method: Theoretical reflection based on literature review. Results: Children and adolescents need to be healthy to learn and have to be taught how to be healthy, thus health and education should work together in order to provide an adequate response to the populations' health and education needs. Given the daily problems that threaten our health and well-being, we should consider the importance of this collaboration. School health is a tool which encompasses not only the health and education system, but also others, such as security forces, civil society, community and family. Conclusions: This is one of the challenges that school and health organizations are facing today: to ensure a proficient relationship with the social context to which they belong, participating and improving school health programs that solve real community needs.
\end{abstract}

Keywords: School health, Health promotion, Equity in health, Education, Community participation. 


\section{Egitania \\ $s$ c i e $\cap$ c i a}

\section{RESUMEN:}

Objetivo: Reflexionar sobre la importancia de la educación para la salud en la promoción de la salud. Método: Reflexión teórica basada en la revisión de literatura. Resultados: Los niños y los adolescentes necesitan estar saludables para aprender y educados para estar saludables, por lo que la salud y la educación deben actuar en conjunto, con el objetivo de dar una respuesta adecuada a las necesidades de salud y educación de las poblaciones. Frente a los problemas cotidianos que amenazan la salud y el bienestar se puede considerar la importancia de esta colaboración, representando la salud escolar un instrumento que abarca no sólo el sistema de salud y la educación, como otros agentes, tales como las fuerzas de seguridad, la sociedad civil, la comunidad y la familia. Conclusiones: Este es uno de los desafíos que enfrentan hoy en día las organizaciones escolares y de salud, garantizar una relación eficaz con el contexto social en que operan, participando y desarrollando programas de salud escolar que satisfagan las necesidades reales de la comunidad.

Palabras clave: Salud escolar, Promoción de la salud, Equidad en salud, Educación, Participación comunitaria.

* Doutor em Educação, Mestre em Ciências da Educação na Especialidade de Educação para a Saúde, Licenciado em Enfermagem. Professor Adjunto. Instituto Politécnico de Portalegre, Portugal. 


\section{Eqitania \\ s c i e $\Omega$ c i a}

\section{INTRODUÇÃO}

As escolas não podem ensinar tudo, as decisões devem ser tomadas sobre o que é mais importante, numa cultura particular, e as opções adequadas devem ser tomadas nesse sentido (Tones, 2005). No entanto, algo que deverá ser globalmente aceite é o facto de que todo e qualquer sistema de educação eficiente deve assegurar um elemento fundamental, a saúde e a qualidade de vida das crianças e dos jovens, promovendo a sua capacidade para aprender. A saúde e a educação são indissociáveis, pois se o estado de saúde está intimamente relacionado com o acesso à escola e com a capacidade de aprender, os comportamentos de saúde estão associados aos resultados escolares, como as avaliações. Estas ligações significam que melhorar a eficácia de um sector pode vir a beneficiar outro sector, sendo as escolas, portanto, um cenário importante para a educação e a saúde (Barnekow et al., 2006).

As condições de salubridade adequadas, associadas a contextos adequados ao desenvolvimento físico, mental e social, são elementos fundamentais para incrementar a adesão escolar e diminuir o absentismo, tornando as instituições de ensino espaços de equidade em saúde, assumindo a responsabilidade de assegurar aos estudantes boas condições neste âmbito e de os preparar para a sua vida futura como adultos e cidadãos. Nesta perspetiva toda a comunidade educativa obtém um maior benefício a este nível, resultante destas condições salutares mais adequadas (Pommier et al., 2009).

O desenvolvimento de programas de saúde escolar adequados, fruto de parcerias de natureza comunitária, será um elemento essencial e sustentável para alcançar um espectro populacional amplo, que vai desde as crianças e adolescentes em idade escolar à própria comunidade em geral, constituindo uma estratégia essencial de promoção de estilos de vida saudáveis. Só desta forma se poderá consolidar um conceito de saúde que vise o desenvolvimento pleno dos indivíduos, grupos e comunidades, numa dinâmica personalizada de cariz cultural, social e económico, sustentado no desenvolvimento de capacidades de integração, participação e assertividade.

O desenvolvimento destes programas permite a edificação de um ambiente escolar mais adequado e saudável, devendo ser objetivo destes projetos a definição de intervenções que possam inclusivamente ser implementadas em contextos com maiores lacunas ao nível dos recursos disponíveis, não sendo apenas direcionados para a prevenção da doença, mas proporcionando aos estudantes a oportunidade de reforçar a sua capacidade de aprender, através da promoção de saúde, física e mental, e do bem-estar social (Percy et al., 2009). 


\section{Egitania}

$s$ c i e $\Omega$ c i a

\section{EDUCAÇÃO E SAÚDE EXPLORANDO NOVAS DIMENSÕES NA SAÚDE ESCOLAR}

As crianças e adolescentes necessitam ser saudáveis para aprender e, consequentemente, educados para ser saudáveis pelo que, mais do que nunca, a saúde e a educação devem continuar a trabalhar em conjunto, nos seus mais diversos níveis e contextos de intervenção, visando proporcionar um adequado e sustentável atendimento às necessidades de saúde e educação desta população-alvo (King \& Eckstein, 2006). Perante os problemas quotidianos que globalmente ameaçam a saúde, pode-se considerar que esta colaboração é cada vez mais importante, representando a saúde escolar um instrumento fundamental, alicerçado num processo de pareceria, que engloba não só o sistema de saúde e de educação, como outros intervenientes, entre os quais se destaca a familia e a comunidade de inserção.

Desta forma, caberá à escola assegurar uma relação eficaz com o contexto social em que se engloba, levando-o a participar nos programas de saúde escolar em que se envolve, mas também envolver-se de forma ativa nos programas desenvolvidos na própria comunidade. Os programas de saúde escolar devem integrar todo um conjunto de ações planificadas, sequenciais e estratégias, abrangendo atividades que poderão ser desenvolvidas por entidades de referência, destinadas a promover o desenvolvimento físico, psicológico, emocional, social e educativo dos estudantes, devendo englobar áreas como a prevenção, educação, cuidados de emergência e gestão de condições de saúde agudas e crónicas, tendo por meta assegurar os cuidados necessários aos estudantes, promover a saúde, identificar e prevenir problemas de saúde e lesões (King \& Eckstein, 2006; Schwab \& Gelfman, 2005).

Para assegurar o seu êxito é indispensável o apoio das familias e da comunidade, que neles devem ser envolvidas, considerando igualmente as necessidades da própria comunidade, os recursos existentes, as regras, normas ou legislação vigentes, devendo os programas ser coordenados por uma equipa multidisciplinar, constituída por diferentes profissionais da área da saúde e da educação, devidamente capacitados para este efeito, que seja responsável pela qualidade das intervenções e pela sua eficácia. Relativamente à sua constituição, importa destacar o conceito de equipa nuclear de saúde escolar, constituída por médicos e enfermeiros a desempenhar funções no âmbito dos cuidados de saúde primários (Ministério da Saúde - Direcção-Geral da Saúde [MS-DGS], 2006).

A estes profissionais podem-se associar outros técnicos de saúde, intervenientes em áreas relevantes ao nível da promoção da saúde, como higienistas orais, técnicos de saúde ambiental, técnicos de serviço social, psicólogos, psicopedagogos, terapeutas da fala, terapeutas ocupacionais, fisioterapeutas, nutricionistas, dentistas, audiologistas, entre outros. Estas equipas multidisciplinares engobam igualmente elementos das organizações escolares, nomeadamente os docentes que exercem funções de coordenação da educação para a saúde e integração das intervenções de saúde escolar nos projetos educativos instituídos, bem como outros que abordem nas suas atividades letivas temáticas englobadas neste 


\section{Eqitania \\ s c i e $\Omega$ c i a}

âmbito. Além dos elementos afetos a organismos da área da saúde e da educação, poderão também integrar estas equipas profissionais oriundos de outras áreas de intervenção, de acordo com as especificidades do contexto e os problemas e necessidades identificadas.

Os programas de saúde escolar têm como principais metas o rastreio, diagnóstico e aconselhamento em saúde, englobando os cuidados de urgência e emergência aos estudantes, a atempada implementação de intervenções adequadas aos problemas de saúde identificados, os exames exigidos e necessários aos estudantes, assistência com medicação durante o período escolar, acompanhamento de saúde das crianças com necessidades especiais e aconselhamento de saúde (MS-DGS, 2006; 2014). Nestes programas encontram-se englobadas atividades de promoção da saúde e prevenção de acidentes, constituindo-se como elementos de ligação e referência relativamente aos diferentes serviços e organizações existentes na comunidade, tendo como principais objetivos:

- Promover a saúde e o bem-estar;

- Prevenir doenças específicas, distúrbios de saúde e lesões;

- Prevenir comportamentos sociais de alto risco;

- Intervir para ajudar crianças e jovens em situação de necessidade ou de risco;

- Apoiar os indivíduos que apresentam necessidades especiais de saúde;

- Promover comportamentos positivos de saúde e segurança (Schwab \& Gelfman, 2005).

Um programa de saúde escolar adequado deverá ser composto por diversos componentes, singulares, mas interligados entre si, que permitam abordar a saúde dos estudantes como um todo, não se dedicando exclusivamente à prevenção da doença, mas tendo como foco principal proporcionar possibilidades de aprendizagem, complementar e sinérgica, fomentando a promoção da saúde (Massachusetts Department of Public Health, 2007). Entre estes componentes, fundamentais para a saúde escolar, evidenciam-se os seguintes:

Educação em saúde: planeamento sequenciado e adaptado às faixas etárias, abordando o desenvolvimento físico, mental, emocional e as dimensões sociais da saúde, desenvolvendo conhecimentos, atitudes e competências, para motivar e auxiliar os alunos a manter e melhorar a sua saúde, prevenir doenças e reduzir os comportamentos de risco relacionados com a saúde;

Educação física: planeamento sequenciado que promova a atividade física ao longo da vida, implementando programas de educação física adequados para desenvolver competências cinestésicas, desportivas e de aptidão física, melhorando as capacitações mentais, sociais e emocionais;

Serviços de saúde: avaliação, proteção e promoção da saúde do estudante, facilitando o atendimento, garantindo o acesso e encaminhamento para a comunidade e outros prestadores de cuidados, promovendo a utilização dos serviços de cuidados de saúde 


\section{Eqitania}

s c i e $\Omega$ c i a

primários, para prevenção, controle de doenças e outros problemas de saúde, prestação de cuidados de emergência, de ensino e de aconselhamento;

Serviços de alimentação: acesso a uma alimentação variada e nutricionalmente adequada, a preços acessíveis, que responda às necessidades de saúde e nutricionais dos estudantes, num ambiente que promova hábitos alimentares saudáveis e a educação nutricional;

Promoção da saúde dos funcionários: manter e melhorar a saúde e o bem-estar do corpo docente e dos funcionários das escolas, que servem como modelos para os estudantes, proporcionando oportunidades de melhoria da sua saúde através de intervenções que incluam avaliações de saúde, educação em saúde e atividade física;

Serviços de aconselhamento psicológico: atividades centradas no domínio cognitivo, emocional, comportamental e social dos indivíduos, grupos e familias, com a finalidade de melhorar a saúde mental, emocional, comportamental e social dos estudantes, proporcionando uma segurança física, um ambiente saudável e de apoio que promova a aprendizagem;

Ambiente escolar saudável: espaços físicos adequados e agradáveis, com uma cultura e clima psicossocial favoráveis, proporcionando um ambiente de apoio, seguro e saudável, que promova a aprendizagem;

Envolvimento da familia e da comunidade: parcerias entre escolas, familias, grupos comunitários e indivíduos, que construam um alicerce amplo de apoio aos esforços dos programas de saúde escolar, reunindo os recursos e os serviços que possam responder eficazmente às necessidades de saúde dos estudantes, numa perspetiva de partilha e maximização de recursos e capacitações que permita o desenvolvimento saudável de crianças e jovens, bom como das suas familias (Percy et al., 2009; King \& Eckstein, 2006; Medical Officers of Schools Association, 1998).

A implementação de programas de saúde escolar implica a criação de parcerias intersectoriais, englobando intervenientes não só das áreas da saúde e da educação como das próprias comunidades, envolvendo de forma ativa e decisiva as crianças e jovens que desenvolvem a sua aprendizagem nas escolas, principais alvos destes processos, com vista a proporcionar-Ihes os conhecimentos e as competências necessárias para obter, gerir e avaliar recursos que contribuam para melhorar a saúde e a qualidade de vida.

Rompendo com as intervenções mais tradicionais desenvolvidas em contexto escolar, no âmbito educação para a saúde, tendo em geral como foco as causas de doença, como o tabagismo, o abuso do álcool e a toxicodependência, o desenvolvimento destas parcerias implica o estabelecimento de estratégias concertadas, que visem transformar as escolas em espaços mais saudáveis, integrando a promoção da saúde de forma global nos currículos, introduzindo programas e práticas saudáveis nas rotinas escolares quotidianas, melhorando as condições de trabalho e promovendo melhores relações, tanto no seu interior como entre elas 


\section{Eqitania \\ $s$ c i e $\Omega$ c i a}

e as comunidades locais (Gillbert, Samyer \& McNeill, 2011). Nesta perspetiva, integra-se uma conceção de promoção da saúde alicerçada num modelo que ultrapassa uma visão meramente assente no binómio saúde-doença, emergindo decisivamente uma orientação de cariz social.

Assumindo que as comunidades escolares respondem a um conjunto dinâmico de fatores que afetam o desempenho dos estudantes e os resultados de aprendizagem, sendo a sua saúde, a dos professores e das familias um fator-chave que influenciam este processo, tornase fundamental a implementação de medidas, neste contexto, que proporcionem um conjunto de princípios e ações para consolidação da saúde, contribuindo as comunidades escolares para a gestão da saúde e das questões sociais, melhorando a aprendizagem dos alunos e a eficácia da escola, em consonância com as prioridades atuais de promoção da saúde, no sentido de fomentar ambientes saudáveis que promovam o desenvolvimento (Barnekow et al., 2006).

O resultado das parcerias intersectoriais, emergentes da própria comunidade, deverá centrarse num conceito de inclusão da tríade escola / comunidade / ambiente, sendo essencial que o contexto em torno da escola reflita os valores nela desenvolvidos, englobando iniciativas comunitárias de apoio que incluam:

- Facultar acessos às escolas seguros e ativos, englobando as redes de transportes públicos e a promoção da segurança rodoviária;

- Restringir a venda e a publicidade de produtos não saudáveis, próximo dos acessos e entradas das escolas;

- Facilitar o acesso a centros sociais para os jovens onde possam apresentar problemas e questões de forma confidencial;

- Proporcionar áreas de lazer atraentes e instalações desportivas na zona de implementação das escolas (Barnekow et al., 2006).

O incremento da saúde escolar tem de ser, cada vez mais, um aspeto integrado na agenda educativa das instituições de ensino, sendo visto como um instrumento que visa contribuir, através de estratégias como a participação, a competência de ação que vise um envolvimento efetivo e sustentado dos membros da comunidade educativa nestes processos e a capacitação para o desenvolvimento democrático, de modo lato, e nas escolas, especificamente, minimizando a desigualdade na distribuição de riscos para a saúde e de oportunidades de aprendizagem nas nossas sociedades (Gillbert et al., 2011). 


\section{Egitania}

$s$ c i e $\Omega$ c i a

\section{AS EQUIPAS DE SAÚDE ESCOLAR COMO ELO DE LIGAÇÃO ESCOLA-COMUNIDADE}

Na saúde escolar, enquanto referencial do sistema de saúde para o processo de promoção e educação para a saúde na comunidade educativa, face ao papel essencial da escola no processo de aquisição de estilos de vida, a intervenção junto das comunidades educativas privilegia estratégias que permitam a gestão das suas condições de saúde, através dos elementos de ligação entre os sistemas de saúde e de educação, as equipas de saúde escolar, devidamente aptas para intervenções neste âmbito (MS-DGS, 2006). Esta ligação, ao nível da educação para a saúde, implica o desenvolvimento de ações nos diferentes níveis de ensino, cobrindo um leque de áreas estratégicas de atuação ao nível de promoção da saúde, em virtude das necessidades da população escolar neste âmbito se tornarem progressivamente mais complexas e exigentes ao longo do ciclo de vida, das quais se pode destacar a saúde individual e coletiva, a inclusão escolar, o ambiente escolar e os estilos de vida.

A operacionalização das atividades desenvolvidas na saúde escolar, nomeadamente dos respetivos programas, depende da conjugação de competências, conhecimentos e empenho de um conjunto de indivíduos, que compõem estas equipas. "As equipas de saúde escolar têm um papel fundamental na sensibilização e reforço das competências dos outros técnicos de saúde, dos pais, dos educadores e dos professores" (MS-DGS, 2006: 30), devendo os agentes responsáveis da promoção da saúde ter conhecimentos sobre os determinantes da saúde e as suas consequências, tanto na vida de cada um como na sociedade em geral.

Estas equipas integram profissionais de várias áreas, mormente da saúde e educação, baseando-se numa conceção multidisciplinar que thes confere a capacidade de responder aos reptos apresentados por estudantes, organizações escolares e a própria comunidade, através de uma ação intersectorial em que a promoção do autocuidado assume um papel crucial, envolvendo estruturas que estimulem e apoiem a construção de projetos futuros, nomeadamente as autarquias, enquanto pólos de incremento da participação comunitária. A sua intervenção implica a recolha de dados e o diagnóstico de situações, identificando problemas que existam no contexto educativo, definindo objetivos individualizados a alcançar e planeando intervenções, desenvolvidas tendo como base as medidas propostas para alcançar os objetivos, que serão implementadas, de acordo com o plano de ação, e posteriormente avaliadas, evidenciando os progressos e os resultados alcançados (King \& Eckstein, 2006; Prazeres, 2002).

Os médicos, enfermeiros e outros técnicos de saúde, que constituem as equipas de saúde escolar, muitas vezes não serão os que normalmente prestam cuidados às familias dos estudantes, podendo inclusivamente não ser aqueles que estas escolheriam para tal. Deste modo, compete a estes profissionais de saúde fazer o possível por ganhar a sua confiança, sendo fundamental construir uma boa relação com o corpo docente das escolas, com os estudantes e com as suas familias, para além de manter uma comunicação e colaboração 


\section{Eqitania \\ s c i e $\Omega$ c i a}

estreita com os seus médicos de familia, envolvendo-os sempre que necessário, para que haja uma efetiva continuidade dos cuidados prestados (Medical Officers of Schools Association, 1998).

Idealmente a equipa de saúde escolar deveria ser responsável pela prestação dos cuidados necessários aos estudantes e funcionários escolares durante o período letivo, no âmbito das competências que são inerentes às suas áreas de intervenção, sendo importante novamente destacar a importância da sua intervenção neste âmbito, facultando o apoio necessário até que estes possam retornar a casa, ao cuidado da sua familia e sob supervisão do seu médico de familia, ou sejam encaminhados para o serviço de saúde adequado à situação em causa, devendo ser comunicado, em ambos os casos, os procedimentos efetuados.

Porque é de educação e de saúde que falamos, o bom será sempre inimigo do ótimo, tornando-se pertinente referir que uma gestão e liderança dos programas de saúde escolar, eficiente e efetiva, constituí um requisito imprescindível para a concretização plena dos objetivos propostos, realçando-se alguns aspetos que se consideram fundamentais para que estes decorram de forma apropriada e com a qualidade preconizada, como o seu adequado financiamento, com uma real adequação dos recursos necessários, uma efetiva administração / gestão dos programas de saúde escolar (o que é efetuado? / quem efetua?), os meios humanos necessários e a definição ética e fundamentada das prioridades e da distribuição de recursos neste âmbito (Wold, 2005).

\section{A SAÚDE ESCOLAR ENQUANTO ELEMENTO MOTRIZ DA PROMOÇÃO DA SAÚDE}

"No final do milénio está a formar-se um novo mundo" (Castells, 2007: 458), despontando uma visão de sociedade que resulta de transformações estruturais que se podem observar nas relações de produção, poder e experiência, que conduzem a mudanças significativas das conceções sociais de espaço e de tempo, motivando a emergência de uma nova cultura de crescimento multidimensional. Aos profissionais das áreas da educação e da saúde o repto que se apresenta implica desenvolver e aplicar metodologias inovadoras de ensinoaprendizagem, que permitam uma melhor comunicação e gestão do conhecimento aproveitando o potencial das tecnologias de informação, possibilitando uma intervenção proactiva ao nível da promoção da saúde na escola, considerando sempre o papel dos próprios estudantes na sua implementação, sendo prioritário desenvolver estratégias ativas e participativas, envolvendo o trabalho interpares, a comunicação interpessoal e a promoção de capacitações de cariz pessoal e social, englobando novas áreas de formação na saúde escolar (MS-DGS, 2006; 2014).

A promoção da saúde na escolar pode, assim, ser definida como qualquer atividade realizada para melhorar e/ou proteger a saúde de todos na comunidade escolar, sendo um conceito 


\section{Eqitania}

s c i e $\Omega$ c i a

mais amplo do que o de educação em saúde, pois engloba atividades relacionadas com políticas escolares saudáveis, o ambiente físico e social da escola, o currículo, as ligações à comunidade e os serviços de saúde (Leger, Young, Blanchard \& Perry, 2010).

As instituições escolares têm a oportunidade única de influenciar decisivamente e positivamente a saúde dos estudantes, mais não seja pelo facto destes passarem a maior parte do seu dia na escola, de segunda-feira a sexta-feira, durante 9 a 10 meses por o ano, participando tanto em atividades letivas como em atividades pós-escolares, nas quais se podem integrar os mais variados aspetos relacionados com a promoção da saúde. Cada vez se torna mais importante aproveitar estas oportunidades, quando se constata existirem evidências de prevalência de determinadas variações comportamentais e de desenvolvimento, progressivamente mais comuns no ambiente escolar (Lewis \& Bear, 2002).

Pode-se então considerar a escola como um microcosmos dos problemas de saúde que ocorrem na comunidade, pois se o tabagismo ou a obesidade forem um problema na população em geral, estarão potencialmente presentes na população escolar, pelo que se houver uma resposta efetiva da comunidade a estes problemas, esta poderá também apresentar resultados positivos em contexto escolar, sendo para tal essencial que os programas de saúde escolar integrem a comunidade e o seu sistema de prestação de cuidados de saúde, no apoio facultado aos estudantes (Massachusetts Department of Public Health, 2007).

No início deste século, os programas de saúde escolar enfrentam desafios sem precedentes, resultantes da multiplicidade de problemas de saúde que podem afetar os estudantes, as suas familias e a própria comunidade, mas também de novas oportunidades para consolidar a sua educação, saúde e qualidade de vida. Face a estas condições societais será pertinente uma reflexão aturada, por parte dos que exercem atividade no âmbito da saúde, que vise procurar resolver algumas questões de carácter formativo, interventivo, ético e legal, a que possam, de alguma forma, ter dado menos atenção anteriormente, a fim de estabelecer uma base sólida, na qual possam edificar a sua prática na atualidade (Costante, 2005).

As mudanças nas normas sociais e as necessidades de saúde crescentes, a par da expansão do conhecimento neste campo, exigem que os programas de saúde escolar evoluam progressivamente, procurando responder às expectativas, que se vão expandido, do papel da escola na vida e na saúde das crianças e jovens, bem como das suas familias. A nível global são múltiplas as importantes mudanças sociais que se vêm verificando, tendo um impacto direto sobre o papel atual dos programas de saúde escolar (Massachusetts Department of Public Health, 2007):

Maior (re)conhecimento do papel da saúde no sucesso educativo: continua a ser evidente o impacto dos problemas de saúde no desempenho escolar dos estudantes, bem como os efeitos positivos duma intervenção atempada na sua resolução, no desenvolvimento presente e futuro, pois é evidente que o sucesso escolar não será compatível com problemas de má 


\section{Eqitania}

s c i e $\Omega$ c i a

nutrição, depressão, insegurança, violência doméstica e abuso de substâncias, que podem prejudicar gravemente a saúde dos estudantes e a sua capacidade de aprender;

Reconhecimento da necessidade das escolas garantir atendimento de crianças com situações de saúde crónicas e com necessidades de saúde especiais: os avanços tecnológicos e da medicina têm associados um aumento da sobrevivência de crianças e jovens com variadas condições médicas e genéticas, às quais se associa o direito à educação, num ambiente menos restritivo do que era advogado anteriormente, que implica a inclusão de estudantes com uma multiplicidade de problemas de saúde nas escolas, exigindo condições de prestação de cuidados de saúde em contexto escolar;

Reconhecimento da importância das escolas como parceiro essencial na implementação de iniciativas de saúde pública: face ao aumento de problemas de saúde pública, as escolas podem desempenhar um papel importante na avaliação e implementação de novas iniciativas, como no caso da vigilância das imunizações programadas ou do excesso de peso, em que podem mudar a oferta nutricional e promover atividades físicas, contribuindo para a incorporação de estilos de vida saudáveis numa idade precoce;

Aumento dos riscos de saúde nas crianças em idade escolar: muitos jovens de hoje correm o risco de problemas de saúde como depressão, violência, abuso sexual, violência doméstica e infeção pelo vírus da imunodeficiência humana, devendo as equipas de saúde escolar estar particularmente atentas, no sentido de procurar identificar estes jovens e facilitar seu acesso ao sistema de saúde, diretamente ou através do encaminhamento para serviços médicos ou sociais apropriados;

Dificuldades económicas e iniquidade no acesso de saúde: as familias em situação de dificuldade económica podem considerar o acesso à saúde apenas essencial em caso de doença, atribuindo uma prioridade menor às consultas preventivas, podendo ainda existir múltiplos obstáculos no acesso aos cuidados como a falta de transporte, a língua diferente, a mobilidade das familias e a insensibilidade dos prestadores de cuidados a condições multiculturais, constituindo a saúde escolar muitas vezes um ponto de entrada acessível para o sistema de cuidados de saúde, ligando o estudante aos serviços prestadores de cuidados de saúde;

Mudanças no padrão de emprego das familias e na estrutura familiar: aumento do número de pais em condições de emprego precário e de familias monoparentais, com dificuldade em justificar ausências no trabalho, que podem tornar por vezes a escola num espaço de triagem ativo de condições de saúde e de lesões comuns, nomeadamente aspetos associados à sexualidade, alimentação, atividade física, comportamentos aditivos e violência em meio escolar;

Mudanças da matriz cultural: os fenómenos migratórios levam à integração de estudantes oriundos de locais diferentes, implicando uma atenção especial na incorporação e adaptação de padrões, valores e atitudes de carácter multicultural; 
Responsabilidade na resposta a emergências da comunidade: perante a possibilidade de ameaças como pandemias ou perturbações sociais, as escolas e os programas de saúde escolar devem estar preparados para estas situações, tendo por base o seu papel na comunidade, enquanto parceiros fundamentais no planeamento e resposta a emergências (Massachusetts Department of Public Health, 2007; MS-DGS, 2014).

Mais do que nunca se constata que os programas de saúde escolar representam uma componente essencial para a segurança e bem-estar das crianças e jovens, sendo a qualidade dessas intervenções um elemento-chave para a sua realização ao longo da vida, no campo educativo e da saúde, numa conceção cada vez mais atual que implica:

- Valorizar a importância da prevenção;

- Promover a aquisição dum pleno potencial para a saúde que permita tomar decisões responsáveis e obter ganhos em saúde;

- Assegurar um atendimento de saúde de qualidade e um ambiente seguro e saudável;

- Construir parcerias para maximizar o acesso a cuidados de saúde equitativos e de qualidade;

- Dedicar especial atenção às necessidades de saúde dos mais carenciados;

- Capacitar as comunidades para se auxiliarem entre si;

- Proteger, preservar e melhorar a saúde da população.

Considerando a necessidade de uma crescente procura dos serviços de saúde escolar, a par de cenários que se perspetivam cada vez mais complexos nas escolas, os paradigmas, as legislações e os regulamentos estabelecidos necessitam ser contestados e revistos, no sentido de que a prestação de serviços de promoção de saúde e de prevenção de acidentes às crianças e jovens se mantenha atualizado com as suas correntes necessidades (Costante, 2005).

\section{CONCLUSÃO}

A efetiva promoção da saúde nas escolas requer que todas as partes envolvidas tenham um manifesto sentido de pertença e participação no processo, representando o trabalho intersectorial e as parcerias instituídas elementos essenciais, sendo os seus principais interessados e intervenientes:

- O sector da educação, incluindo escolas e professores;

- O sector da saúde e os serviços de promoção da saúde;

- Os estudantes;

- Os investigadores em promoção da saúde (Barnekow et al., 2006; Green \& Tones, 2010). 


\section{Eqitania}

s c i e $\Omega$ c i a

Os conceitos nos quais se baseiam os programas de saúde escolar não são novos, pois há mais de 150 anos que é reconhecida a importância da saúde na educação e o papel crítico desempenhado pelos programas de saúde escolar. Com base nos conhecimentos e experiências adquiridas, cabe às equipas de saúde escolar e aos agentes de promoção da saúde uma missão complementar e de apoio à escola, na sua função primordial de promover e melhorar a saúde dos estudantes, que se reflete nos seguintes aspetos (Massachusetts Department of Public Health, 2007):

Resposta a necessidades cada vez maiores: o sucesso de integração de crianças com necessidades de saúde especiais e os esforços de expansão da cobertura da educação préescolar têm resultado num maior número de crianças em idade escolar;

Aumento da eficácia de outros investimentos em desenvolvimento infantil: os programas de saúde escolar são a sequela e o complemento mais essencial aos programas de cuidados e de desenvolvimento infantil;

Consolidação do aproveitamento escolar: assegurar uma boa saúde em idade escolar incita a frequência escolar, reduz as reprovações e aumenta o sucesso escolar;

Melhoria da equidade social: as crianças oriundas de meios mais desfavorecidos irão beneficiar de intervenções na área da saúde, sendo provável que demonstrem melhorias substanciais na frequência e nos resultados escolares;

Estratégia altamente eficaz em termos de custos: os programas de saúde escolar contribuem para a ligação entre recursos da saúde e da educação, numa infraestrutura - a escola - que já existe, que é penetrante e que é sustentável.

Ao nível da promoção da saúde escolar o desafio futuro, para as organizações envolvidas neste âmbito, será incentivar o desenvolvimento das relações entre os profissionais das escolas e da comunidade, independentemente de se tratarem ou não de profissionais de saúde, garantindo uma aproximação que promova globalmente a saúde, para além do necessário e contínuo desenvolvimento das competências para estabelecer uma cultura e perspetiva comum entre as áreas da saúde e da educação, dentro e fora da escola (Pommier et al., 2009).

Numa conceção em que as escolas utilizem as suas próprias estruturas edificadas, as suas relações internas e externas, as suas estratégias de ensino-aprendizagem e o seu potencial de criação de sinergias com o contexto social envolvente, estamos perante um conjunto de condições que possibilita criar os meios adequados de promoção da saúde, melhorando a saúde física e emocional de todos os envolvidos na comunidade escolar e comunidade envolvente, sendo esta uma motivação essencial subjacente a estes processos.

Em síntese, poderão ser considerados como elementos fundamentais para a promoção da saúde em meio escolar a definição de políticas de escolas saudáveis, o ambiente físico da escola, o ambiente social da escola, as competências individuais de saúde e competências 


\section{Eqitania \\ $s$ c i e $\Omega$ c i a}

para a ação, a ligação à comunidade e os serviços de saúde (Leger et al., 2010; Green \& Tones, 2010). Nesta perspetiva abraça-se o desafio fundamental que a educação e promoção da saúde atualmente enfrentam, fomentar a participação da comunidade, considerando que o potencial dos indivíduos para refletir e agir de forma construtiva na identificação e resolução dos seus problemas de saúde, bem como dos que emergem no grupo e comunidade que integram, constitui no quotidiano um elemento essencial, permitindo a sua autossuficiência no desenvolvimento de capacitações e competências que os tornem membros ativos e de direito na promoção da saúde.

\section{REFERÊNCIAS BIBLIOGRAFICAS}

Barnekow, V., Buiss, G., Clift, S., Jensen, B., Paulus, P., Rivett, D. et al. (2006). Health-promoting schools: A resource for deVeloping indicators. Copenhagen: International Planning Committee of the European Network of Health Promoting SChools.

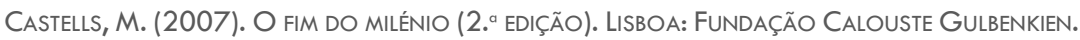

Costante, C. (2005). Future Challenges for school health services and the laW: A manager's perspective. In Schwab, N. \& Gelfman, M. (EDS.), LEGAL ISSUES IN SCHOOL HEALTH SERVICES: A RESOURCE FOR SCHOOL ADMINISTRATORS, SCHOOL ATTORNEYS, SCHOOL NURSES (PP. 489 507). LINCOLN: IUNIVERSE.

Gilbert, G., Sawyer, R. \& MCNeill, E. (2011). Health education: Creating strategies for school and community health (3.rd EDITION). SUDBURY: JONES AND BARTLETT PUBLISHERS.

Green, J. \& Tones, K. (2010). Health promotion: Planning and strategies (2.nd edition). London: Sage Publications.

King, D. \& Eckstein, J. (2006). Manual for school health programs. Jefferson City: Missouri Department of Elementary and Secondary Education - Missouri Department of Health and Senior Services.

Leger, L., Young, I., Blanchard, C. \& Perry, M. (2010). Promoting health in schools: From evidence to action. Saint Denis Cedex: International Union for Health Promotion and Education.

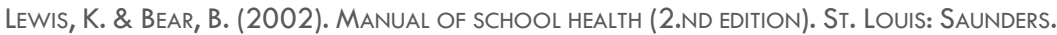

Massachusetts Department of Public Health (2007). Comprehensive school health manual (2.nd edition). Boston: Massachusetts Department of Public Health.

Medical Officers of SChools Association (1998). Handbook of SChOOl health (1 8.th edition). StaffordShire: Trentham Books.

Ministério da Saúde - Direcção-Geral da Saúde [MS-DGS] (2014). Programa nacional de Saúde escolar. Lisboa: Direcção-Geral DA SAÚDE.

Ministério da Saúde - Direcção-Geral da Saúde [MS-DGS] (2006). Programa nacional de saúde escolar. Lisboa: Direcção-Geral DA SAÚDE.

Percy, M., MORTenson, J., Labuski, C., Lipani, M., Anderson, A., Boski, E. et Al. (eds.) (2009). TeXas guide to school health programs. austin: Texas Department of State Health Services - Child Health and Safety Branch: School Health Program.

Pommier, J., Jourdan, D., Berger, D., Vandoorne, C., Piorecka, B. \& Carvalho, G. (2009, Agosto). School health promotion: Organization of services and roles of health professionals in seven European countries. The European Journal of Public Health ADVANCE ACCESS, $1-7$.

Prazeres, V. (2002). Saúde dos adolescentes. In Silva, L. (ORG), Promoção da Saúde (Pp. 117 -1 140). Lisboa: Universidade Aberta. 


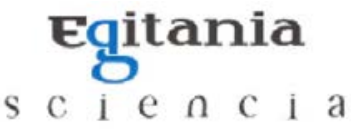

SChWAB, N. \& GelfmaN, M. (EDS.) (2005). Legal ISSUES IN SCHOOl HEALTH SERVICES: A RESOURCE FOR SCHOOL ADMINISTRATORS, SCHOOL ATTORNEYS, SCHOOL NURSES. LINCOLN: IUNIVERSE.

Tones, K. (2005). Health promotion in SChools: The radical imperative. In Clift S. \& Jensen B. (eds.), The health promotion SChool:

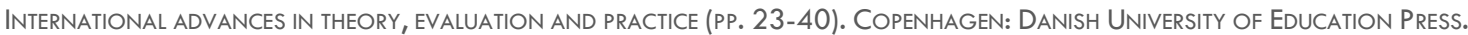

Wold, S. (2005). SChool health Services: history and trends. In SChWAB, N. \& Gelfman, M. (eds.), Legal issues in SChoOl health SERVICES: A RESOURCE FOR SCHOOL ADMINISTRATORS, SCHOOL ATTORNEYS, SCHOOL NURSES (PP. 7-54). LINCOLN: IUNIVERSE. 